\title{
Herausforderung Europa
}

\section{Professionell und vernetzt - die wohlfahrtsverbandliche Soziallobby}

\author{
Günter Rieger
}

Prof. Dr. Günter Rieger leitet die Grundausbildung im Bereich Sozialwesen an der Berufsakademie Stuttgart.

E-Mail grieger@BA-Stuttgart.de
Die deutschen Wohlfahrtsverbände betreiben in der Europäischen Union einen durchaus erfolgreichen Lobbyismus. In zablreichen Netzwerken sind sie eingebunden und binden andere ein. Allerdings entsteht durch die fortschreitende Professionalisierung des Verbandslobbyings ein Demokratiedefizit: Die direkte Beteiligung von Betroffenen und ibrer Selbsthilfeorganisationen bleibt defizitär.

Sozialpolitik ist in Europa weiterhin eine weitgehend nationale Angelegenheit. Ein europäischer Sozialstaat existiert nicht und ist politisch auch nicht gewollt. Die Mitgliedsstaaten folgen unterschiedlichen sozialpolitischen Traditionen. Liberale, konservative und sozialdemokratische Wohlfahrtsstaatstypen (vgl. Esping-Andersen 1998) differieren hinsichtlich Gewichtung, Umfang und Finanzierung der sozialen Sicherung (BMAS (Hg.) 2006; Kaufmann 2003; Schmid 2002). Organisation und Erbringung sozialer Dienstleistungen unterscheiden sich erheblich (Puhl 1997).

Der Prozess der europäischen Einigung wurde zunächst vorrangig durch ökonomische Zielvorgaben bestimmt. Im Kern ging es um die Schaffung eines gemeinsamen Marktes mit freiem Waren-, Kapital-, Personen- und Dienstleistungsverkehr. Soziale Belange wurden dabei nur insofern berücksichtigt, als sie ökonomisch notwendig und nützlich erschienen. Dies galt für die frühen Bemühungen um die Gleichstellung von Mann und Frau ebenso wie für den mit der Freizügigkeit der Arbeitnehmer verbundenen sozialrechtlichen Regelungsbedarf.

Spätestens seit Ende der 1980er Jahre ist dann allerdings eine stärker sozialpolitische Ausrichtung des Integrationsprozesses zu beobachten: 1989 wird die Charta der Grundrechte der Arbeitnehmer (Sozialcharta) verabschiedet; 1993 begründet der Vertrag von Maastricht zur Europäischen Union (EU) mit dem Protokoll zur Sozialpolitik eine gemeinsa- me Arbeitsmarkt- und Beschäftigungspolitik; schließlich erweitert 1999 der Vertrag von Amsterdam den rechtlichen Spielraum für eine eigenständige europäische Sozialpolitik. Er bildet die Grundlage für die mit der Lissabonner Strategie (März 2000) angestrebte Abstimmung der Wirtschafts-, Beschäftigungs- und Sozialpolitik der EU und die sozialpolitischen Agenden I (2000 - 2005) und II (2005 - 2010).

\section{Europa als neue Arena der Sozialarbeitspolitik}

Die sozialpolitischen Maßnahmen der Europäischen Union zielen vor dem Hintergrund eines verschärften globalen Wettbewerbs sowie tiefgreifender Prozesse demografischen und sozialen Wandels in den Mitgliedsstaaten auf eine Modernisierung des europäischen Sozialmodells. Es geht um die Harmonisierung und Weiterentwicklung sozialer Sicherung unter Berücksichtigung der gewachsenen Strukturen in den Mitgliedsstaaten (Subsidiaritätsprinzip). Es gilt Sozialdumping zu verhindern und soziale Mindeststandards zu garantieren. Als Aufgabenschwerpunkte werden (1) eine nachhaltige Beschäftigungspolitik, (2) Chancengleichheit, (3) die Bekämpfung der Armut und Förderung der sozialen Eingliederung sowie (4) Nichtdiskriminierung genannt.

Zur Umsetzung einer europäischen Sozialpolitik steht ein differenziertes Instrumentarium zur Verfügung: (1) Rechtsvorschriften (Verordnungen, Richtlinien, Entscheidungen und Empfehlungen/Stellungnahmen), (2) finanzielle Förderung von Projekten für spezifische Problemgruppen und strukturschwache Regionen (insbesondere Europäischer Sozialfonds [ESF] und spezifische Aktionsprogramme [DAPHNE, Jugend in Aktion usw.]), (3) Konsultationsinstrumente (Mitteilungen, Grünbücher, Weißbücher), (4) sozialer 
Dialog (Einbeziehung der Sozialpartner insbesondere der Arbeitgeberverbände und Gewerkschaften in sozialpolitische Entscheidungen), (5) Mainstreaming (die systematische Hinterfragung aller Maßnahmen aus einer spezifischen Perspektive (z. B. Gender-Perspektive)) und (6) die "Methode der offenen Koordinierung « (MOK) (vgl. Rieger 2008).

Die direkte wie indirekte Beeinflussung der nationalen Sozialpolitiken, die zunehmende Regelungsdichte und der wachsende Förderumfang europäischer Sozialpolitik fordern die Organisationen Sozialer Arbeit heraus. Einerseits werden Handlungsrahmen, Qualität und Professionalisierung Sozialer Arbeit durch Regelungen der Europäischen Union gravierend beeinflusst (z. B. Dienstleistungsfreiheit, Qualifikationsniveaus usw.). Andererseits eröffnen die EU-Fördertöpfe neue Spielräume der Projektfinanzierung zur Entwicklung Sozialer Arbeit. Wer die Handlungsbedingungen Sozialer Arbeit gestalten will, muss die europäische Ebene der Sozial(arbeits)politik in Blick nehmen. Es gilt, die Interessenvertretung Sozialer Arbeit in eigenem und anwaltlichen Interesse zu intensivieren sowie zeitnah, präzise Informationen über Fördermittel, Förderrichtlinien und Antragswege zur Verfügung zu stellen.

\section{Einflusschancen für gut organisierte, ressourcenstarke Interessengruppen}

Das Soziallobbying muss sich auf die spezifischen Gegebenheiten der europäischen Politikarena einstellen. Hier gelten andere Regeln als auf nationaler Ebene. Gewachsene korporatistische Strukturen fehlen, die Entscheidungsprozesse sind äußerst komplex und nach wie vor dem Wandel unterworfen. Europäisches Regieren wird zwischen Rat, Kommission und Europarlament gestaltet, erfordert die Einbindung der jeweiligen nationalen Regierungen und Ministerialbürokratien in das EU-Mehrebenensystem und verlangt die Beteiligung der bislang national integrierten Zivilgesellschaft. Hierarchische Steuerung ist hier noch viel weniger möglich als auf nationaler Ebene. "Governance in der Europäischen Union « ist ein Regieren in Netzwerken (Jachtenfuchs/Kohler-Koch 2004).

Gerade die Komplexität des Verfahrens, das Fehlen oder die Vervielfältigung dominanter politikfeldspezifischer Traditionen und parteipolitischer Versäulung sowie die daraus resultierende notwendige Offenheit für mannigfaltige nationale Besonderheiten und Interessen verursachen einen hohen Beratungsbedarf und eröffnen vielfältige Einflusschancen für gut organisierte Interessenvertretung. Hier spielt die »Champions League des Lobbyismus «. Die Europäische Union ist ein ebenso notwendiges wie »unwider- ten etc., die alle mit ihrer unterschiedlichen Herkunft in Zusammenhang stehen. Sie müssen sich miteinander arrangieren, versuchen aber zugleich im Wettbewerb untereinander, Unterstützung sowohl von innerhalb als auch von außerhalb ihrer Organisationen zu erhalten. Sie öffnen ihre Türen für Informationen und Unterstützung durch jegliche Art von öffentlichen oder privaten Interessengruppen, von nationalen Ministerien bis

\section{"Entgegen mancher Vorurteile ist das politische System der Europäischen Union vergleichsweise offen und transparent "}

stehliches Betätigungsfeld für Lobbyisten « (von Schendelen 2006: S. 135). Notwendig, weil der Einfluss europäischer Rechtssetzung auf die nationale Gesetzgebung immer größer wird und die Attraktivität unterschiedlicher Förderprogramme zunimmt. Unwiderstehlich, weil das politische System der Europäischen Union »vergleichsweise offen und transparent « (ebd. S. 137) ist.

Offenheit und Transparenz folgen aus der Heterogenität und (für Insider) Überschaubarkeit des Systems. In den Institutionen der Europäischen Union handeln Menschen mit ganz unterschiedlichen »Werten, Normen, Interessen, Loyalitä- hin zu kommunalen Mitarbeitern und von Unternehmen bis zu NGOs.« (Ebd. S. 135)

Dafür müssen sie im Gegenzug ihre Vorhaben publik machen und entsprechende Informationen verbreiten. Markantes Beispiel sind hier die regelmäßigen "Aufrufe zur Interessenbekundung « (»calls for interests «) durch die Kommission. Aber auch durch »die frühzeitige Publikation von Tagesordnungen, Protokollen, Entwürfen und weiterer Informationen über Vorhaben, die sich in der Planungsphase befinden, ist die Arbeit von Kommission, Parlament und sogar des Ministerrats transparenter als die seiner

\section{Europa im Internet}

Europäische Kommission: http://www.europa.eu.int Europäisches Parlament: http://www.europarl.eu.int

Rat der Europäischen Union: http://www.ue.eu.int/de/summ.htm

Wirtschafts- und Sozialausschuss: http://www.eesc.europa.eu.int

Ausschuss der Regionen: http://www.cor.europa.eu/de/index.htm

Europäischer Bürgerbeauftragter:

http://www.euro-ombudsman.eu.int/home/de/default.htm

Europäisches Armutsnetzwerk: http://www.eapn.org

Europäische Runde Tisch der Wohlfahrtsverbände: http://www.etwelfare.com

Europäische soziale Nicht-Regierungsorganisationen:

http://www.socialplatform.org

Eurodiaconia: http://www.eurodiaconia.org

Observatorium für die Entwicklung Sozialer Dienste in Europa (ISS/DV):

http://www.soziale-dienste-in-europa.de

Zusammenstellung Günter Rieger 


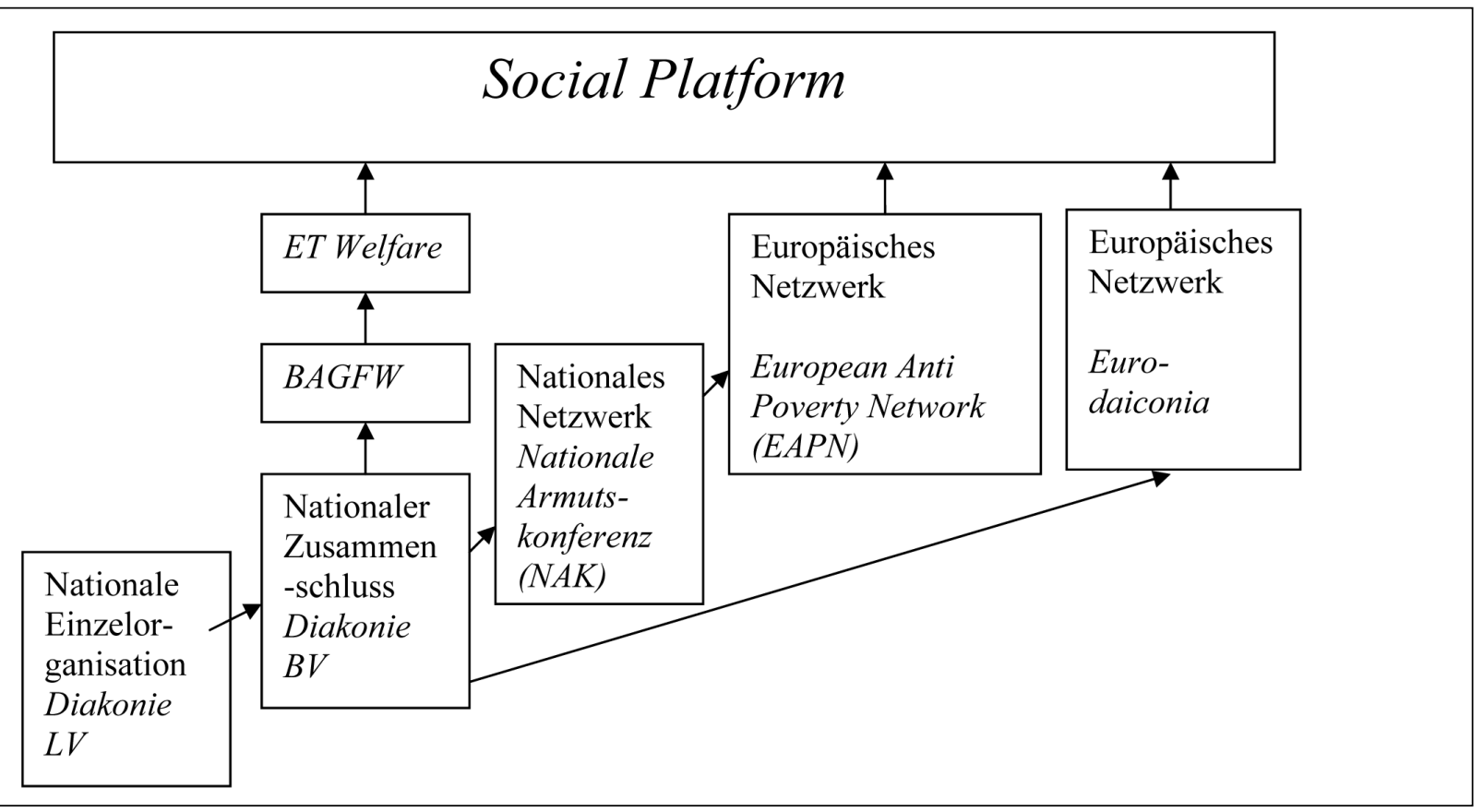

Grafik: Verknüpfungsstruktur Sozialer Nicht-Regierungs-Organisationen am Beispiel der Diakonie

funktionalen Äquivalente in den meisten Mitgliedsstaaten « (ebd.136).

Schließlich sind die Arbeitseinheiten der Kommission, obwohl vielfach als bürokratische Moloche kritisiert, vergleichsweise klein und überschaubar. »Im Jahre 2005 beschäftigte die Kommission rund 14.000 Beamte für die inhaltliche politische Arbeit ... Der Beamtenapparat ist damit kleiner als derjenige vieler Ministerien in den Mitgliedsstaaten. Im Durchschnitt beschäftigen die rund 25 Generaldirektionen (GD; eine Art Mini-Ministerium) ... jeweils etwa 400 Mitarbeiter. Die internen Policy Units die z. B. an ... Programmen der sozialen Absicherung arbeiten, bestehen aus etwa 20 Personen « (ebd.). Die Zahl der handelnden Personen bleibt überschaubar, während das große Arbeitspensum die "Chefs de Dossier « von Policy Units dazu zwingt, sich »bei der Erarbeitung von Grünbüchern (welche >das Problem< definieren), Weißbüchern (welche >die Lösung ‘ des Problems präsentieren) und den konkreten Vorschlägen für Rechtsakte« externen Rats $\mathrm{zu}$ bedienen.

\section{Professionalisiertes, vernetztes Soziallobbying}

Die Spitzenverbände der Freien Wohlfahrtspflege reagieren auf die europäische Herausforderung und ihre Chancen, indem sie ihr Soziallobbying professionali- sieren und Netzwerke bilden. Sie installieren Europareferenten und Fachstellen, um die Interessenvertretung in den relevanten Gremien der Europäischen Union (Ministerrat, Europäisches Parlament, Kommission, Wirtschafts- und Sozialausschuss usw.) wie auf nationaler Ebene (Parlamentsausschüsse und Ministerien in Bund und Ländern) zu gewährleisten und forcieren den Zusammenschluss von Nichtregierungsorganisationen (NGO) in transnationalen, europaweiten Vereinigungen und Netzwerken (Europäisches Armutsnetzwerk; Europäische Runde Tisch der Wohlfahrtsverbände usw.). Ziel ist es bereitgestellte Ressourcen optimal zu nutzen und einen stärkeren Einfluss auf die sozialpolitische Gestaltung des europäischen Integrationsprozesses zu nehmen.

Insbesondere die kirchlichen Verbände Caritas und Diakonie verfügen über eine ausgebaute Infrastruktur zur direkten Vertretung sozialer Interessen auf europäischer Ebene. Sie unterhalten nicht nur auf nationaler Ebene (Landes-, Diözesanoder Bundesebene) entsprechende Europareferate, sondern sind mit eigenständigen Vertretungen in Brüssel präsent und in einer Vielzahl verbandsübergreifender und transnationaler Netzwerke eingebunden. Die Vielfalt der Organisationsformen verbandlicher sozial(arbeits)politischer Interessenvertretung in Europa soll hier am Beispiel des Diakonischen Werkes der Evangelischen Kirche in Deutschland dargestellt werden.
Das Diakonische Werk verfügt in Brüssel über eine eigenständige Repräsentanz in Form der Dienststelle Brüssel als Niederlassung des Diakonischen Werks der Evangelischen Kirchen Deutschlands e. V. und des EuropabüroDiakonieRegional (der Diakonischen Werke Rheinland, Westfalen und Lippe) (EDR). Während die Dienststelle Brüssel im Wesentlichen klassische Lobbyarbeit leistet, ist das EuropabüroDiakonieRegional auf Serviceleistungen zur Information, Beratung und Vertretung spezialisiert.

Zur Lobbyarbeit der Niederlassung des Diakonischen Werkes der EKD gehört (1) ein intensives Monitoring der sozialpolitisch relevanten Politikprozesse im Sinne eines Frühwarnsystems: Welche Themen werden diskutiert? Welche sozialpolitischen Ziele kristallisieren sich heraus? Welche Entscheidungen stehen an? Sodann finden (2) die üblichen Techniken der Interessenvertretung Anwendung: Stellungnahmen und Gutachten in Konsultationsprozessen auf der Arbeitsebene der EU-Kommission, Teilnahmen bei Anhörungen im Europäischen Parlament, Organisation von Fachgesprächen und Tagungen, Jahresempfänge, informelle Kontakte, Lobbybriefe usw. Dabei ist (3) die generelle Strategie und Vorgehensweise mit der Verbandszentrale in Deutschland (Berlin/Stuttgart) abzustimmen; einerseits um die strategischen und inhaltlichen Ziele der Lobbyarbeit festzulegen und andererseits um die Lobbyar- 
beit in der Europäischen Union durch entsprechende Einflussnahme auf die nationale Regierung zu flankieren und so indirekten Einfluss zu organisieren.

Dagegen versteht sich das EuropabüroDiakonieRegional in erster Linie als Serviceagentur für ihre Mitglieder. Gegründet und unterhalten durch die finanz- und mitgliederstarken Diakonischen Werke Rheinland, Westfalen und Lippe werden seine Angebote inzwischen flächendeckend durch Diakonische Werke in der Bundesrepublik in Anspruch genommen. $\mathrm{Zu}$ seinen Leistungen zählen: das "Infogramm « als Informationsdienst für Leitungskräfte, die Erschließung von Förderprogrammen für kirchliche und diakonische Arbeit (Förderbedingungen, Antragsfristen, Begleitung bei der Antragstellung), EUBase als aktuelle Datenbank für EU-Programminformationen (www.eubase.de - Datenbank der Evangelischen Banken zu europäischen Förderprogrammen), die Repräsentation regionaler diakonischer Interessen und Netzwerkarbeit mit den Informations- und Verbindungsbüros anderer Organisationen und der Landesvertretungen (der Bundesländer) in Brüssel.

Daneben fließen die Interessen des Diakonischen Werkes in die Arbeit des Europabüros der Bundesarbeitsgemeinschaft der Freien Wohlfahrtspflege (BAGFW) ein. Das Europabüro der BAGFW vertritt den Zusammenschluss der sechs Spitzenverbände der Freien Wohlfahrtspflege in Deutschland - Arbeiterwohlfahrt, Caritasverband, Deutsches Rotes Kreuz, Diakonie, Paritätischer Wohlfahrtsverband, Zentralwohlfahrtsstelle der Juden - gegenüber den Institutionen der Europäischen Union. Insbesondere hat die Bundesarbeitsgemeinschaft der Freien Wohlfahrtspflege Sitz und Stimme im Europäischen Wirtschafts- und Sozialausschuss, einem beratenden Organ der Europäischen Union, in welchem Arbeitgebervertreter, Arbeitnehmervertreter und andere Interessengruppen eingebunden sind. Als Gründungsmitglied ist die Bundesarbeitsgemeinschaft der Freien Wohlfahrtspflege wiederum Teil des European Round Tabel of Charitable Social Welfare Associations (ETWelfare), der als europäisches Netzwerk mitgliedsstaatlicher Wohlfahrtsverbände die Interessen gemeinnütziger Anbieter sozialer Dienstleistungen wahrnimmt.

\section{Netzwerke von Netzwerken}

Schließlich ist das Diakonische Werk und diakonische Organisationen Mitglied in diversen transnationalen Netzwerken Sozialer Arbeit in Europa (vgl. Hamburger u. a. 2002). Grundsätzlich gilt, dass mit Bezug auf die Organisationen Sozialer Arbeit eine zunehmende Vernetzung auf europäischer Ebene zu beobachten ist. Dies ist eine politisch gewollte und geförderte Entwicklung. "Lokale und regionale einerseits und europäische Vernetzung andererseits wird zum operativen Element des europäischen Sozialmodells. Die Netzwerkarbeit auf europäischer Ebene wird von politischer Seite zum strategischen Instrument der Sicherung von Innovation und Effektivität und zur Realisierung der >offenen Methode der Koordinierung . So wurde die Förderung der Netzwerkarbeit der verschiedenen Akteure Sozialer Arbeit als eigene Gemeinschaftsaufgabe der Kommission definiert. « (Ebd. S. 3 f.) Dabei geht es nicht allein um die Weiterentwicklung der Sozialen Arbeit selbst, sondern ebenso um ihre Politik steuernde und gestaltende Funktion.

Die Diakonie ist jenseits vielfältiger informeller Kontakte und Austauschmöglichkeiten in unterschiedlichen Netzwerken engagiert, die den Charakter von Allianzen haben. $\mathrm{Zu}$ berücksichtigen sind hier Eurodiaconia, als dem europäischen Verband der Diakonie und die European Platform of Social NGOs (Social Platform), als ein transnationales Netzwerk von Organisationen Sozialer Arbeit. Zu Eurodiaconia haben sich 1996 protestantische Kirchen, freie Wohlfahrtseinrichtungen und ökumenische Non-Profit-Organisationen zusammengeschlossen. Eurodiaconia hat aktuell 32 Mitglieder. Sie fördert Wissensaustausch und Zusammenarbeit ihrer Mitglieder, bringt Projekte und Partner zusammen und beteiligt sich an der sozialpolitischen Diskussion in Europa. Sie unterhält ihrerseits Beziehungen zu einer Vielzahl anderer zivilgesellschaftlicher Netzwerke und Organisationen, die sich für ein solidarisches und gerechtes Europa einsetzen. Dazu gehören: Caritas Europa, EAPN (European Anti Poverty Network), European Platform of Social NGOs (Social Platform), Churches Commission on Migrants in Europe (CCME),
Konferenz Europäischer Kirchen (KEK), der Europäische Verband protestantischer Entwicklungsorganisationen (APRODEV) u. a. m.

Die Social Platform ist ein Netzwerk von Netzwerken Sozialer Organisationen. Initiiert (1993) unter Federführung des Europäischen Armutsnetzwerkes (EAPN) wurde und wird die Institutionalisierung dieses Netzwerks massiv durch die Europäische Kommission unterstützt. Die Social Platform besteht aus 38 festen Mitgliedsorganisationen (vgl. Annual Report 2006) und hat eine Reihe so genannter assoziierter Mitglieder und Beobachter. Sie verfügte 2006 über einen Etat von 890.616 Euro, der zu circa 75 Prozent aus Fördermitteln der Europäischen Kommission stammte (vgl. ebd.). Ziele der Social Platform sind

- »Einflussnahme auf die Politikformulierung und Implementation

- Aufbau und Pflege der Kooperation zwischen sozialen NGO und den Institutionen der EU mit dem Ziel der Stärkung des zivilen Dialogs

- Aufbau und Pflege der Kooperation der sozialen NGO, Förderung des Erfahrungs- und Informationsaustauschs

- Formulierung gemeinsamer Positionen unter Respektierung der individuellen Ziele ihrer Mitglieder

- Sensibilisierung und Mobilisierung der Öffentlichkeit « (Hamburger 2002: S. $15)$.

Die Social Platform gestaltet sich als ein besonders dichtes, vielfach verschachteltes Netzwerk von Netzwerken. Durch diese Organisationsform gelingt es ihr »über 1.700 einzelne Organisationen « (ebd. S. 12) einzubeziehen. Wie sich dieses mehrstufige Netzwerk von Netzwerken zusammensetzt, lässt sich für die Diakonie gut am Beispiel des europäischen Armutsnetzwerkes (EAPN) als einem bedeutsamen (Gründungs-) Akteur innerhalb der Social Platform beobachten (vgl. Grafik Seite 109). Landesverbände und Bundesverband der Diakonie sind über die nationale Armutskonferenz Teil der EAPN, welche wiederum Mitglied der Social Platform ist. Gleichzeitig ist das Diakonische Werk über den transnationalen Zusammenschluss protestantischer Organisationen vertreten, denn Eurodiaconia ist selbst eine der 38 Mitgliedsorganisationen der Social Platform. Schließlich ist die Diakonie als Spitzenverband der freien Wohlfahrtspflege auch über 
den europäischen Runden Tisch für Wohlfahrtspflege (ETWelfare) und die Bundesarbeitsgemeinschaft der Freien Wohlfahrtspflege eingebunden.

\section{Resümee}

Enge Vernetzung und Kooperation der auf europäischer Ebene agierenden Organisationen Sozialer Arbeit und die Professionalität des Lobbyings scheinen gleichermaßen Voraussetzungen für erfolgreiche Einflussnahme:

- Das jeweilige Politikfeld muss hinsichtlich der Politikinhalte wie der Positionierung möglicher Gegner, Konkurren- ten oder Verbündeter sorgfältig beobachtet werden (Monitoring).

- Das jeweilige Soziallobbying ist strategisch zu planen und muss auf Kontinuität hin angelegt sein; verfügbare Lobbytechniken sind einzusetzen und die fachliche Qualität von Stellungnahmen und Gutachten muss gewährleistet sein. Die Wohlfahrtsverbände betreiben auf diese Weise einen durchaus erfolgreichen Beschaffungs- und Gesetzeslobbyismus. Die fortschreitende Professionalisierung des Verbandslobbyings verweist aber auf ein Demokratiedefizit. Die direkte Beteiligung von Betroffenen und ihrer Selbsthilfeorganisationen (insbesondere aus dem Kreis der sogenannten Rand- gruppen) bleibt defizitär. Angesichts der Kosten, des Organisationsaufwands und des erforderlichen Know-hows bleiben sie weitgehend auf die advokatorische Vertretung ihrer Interessen durch die Verbände angewiesen. Diese bleiben herausgefordert ihre Doppelfunktion als Anwalt sozialer Interessen und Lobbyist in eigener Sache zu legitimierten. Auch Partizipationsförderung gehört zu ihrem professionellen Mandat.

\section{Literatur}

Benz, A. (Hg.) 2004: Governance. Regieren in komplexen Regelsystemen. Eine Einführung, Wiesbaden.

Bundesministerium für Arbeit und Soziales (Hg.) 2006: Sozial-Kompass EUROPA. Soziale Sicherheit in Europa im Vergleich, Berlin (CD-ROM).

Däubler, W. 2006: Die Europäische Union als Wirtschaftsund Sozialunion, in: Weidenfeld, W. (Hg.), S. 273-288.

Dagger, S./Kambeck, M. (Hg.) 2007: Politikberatung und Lobbying in Brüssel, Wiesbaden.

Esping-Anderson, G. 1998: Die drei Welten des Wohlfahrtskapitalismus, in: Lessenich, S./Ostner, I. (Hg.): Welten des Wohlfahrtskapitalismus: Der Sozialstaat in vergleichender Perspektive, Frankfurt am Main, S. 19-58.

Hamburger, F. u. a. 2002: Strickwerk oder Strategie. Netzwerke der Sozialen Arbeit in Europa. Arbeitspapier Nr. 9 (Observatorium für die Entwicklung der Sozialen Dienste in Europa (ISS)), Frankfurt am Main.

Herrmann, P. 2005: Sozialmanagement in Europa. Herausforderungen verstehen, Strukturen kennen, Vorteile nutzen, Baden-Baden.

Jachtenfuchs, M./Kohler-Koch, B. 2004: Governance in der Europäischen Union, in: Benz, A. (Hg.), Wiesbaden, S. 77102.

Kaufmann, F.-X. 2003: Varianten des Wohlfahrtstaats, Frankfurt am Main.

Kleinfeld, R. u. a. (Hg.) 2007: Lobbying. Strukturen - Akteure - Strategien, Wiesbaden.

Kowalsky, W. 1999: Europäische Sozialpolitik. Ausgangsbedingungen, Antriebskräfte und Entwicklungspotenziale. Lehrtexte. Grundlagen für Europa, Bd. 4, Opladen.

Kropp, S. u. a. (Hg.) 2006: Sozialraum Europa. Sozialpolitik in der erweiterten Europäischen Union. Ein Werkstattbericht, Münster.

Lange, C. 2001: Freie Wohlfahrtspflege und europäische Integration. Zwischen Marktangleichung und Sozialer Verantwortung. Diss. Europäische Sozialpolitik (EU) Bd. 1 IV, Verlag Soziale Theorie und Praxis.
Leif, T/Speth, R. (Hg.) 2006: Die fünfte Gewalt. Lobbyismus in Deutschland, Bonn.

Linzbach, C./Lübking, U. (Hg.) 2005: Die Zukunft der sozialen Dienste vor der Europäischen Herausforderung, BadenBaden.

Matyja, M. 2007: Interessenverbände im Entscheidungsprozess der Europäischen Union, in: Kleinfeld, R. u. a. (Hg.), Wiesbaden, S. 148-168.

Michalowitz, I. 2007: Lobbying in der EU, Wien.

Platzer, W. 2006: Interessenverbände und europäischer Lobbyismus, in: Weidenfeld, W. (Hg.), S. 186-202.

Puetter, U. 2007: Die Wirtschafts- und Sozialpolitik der EU, Wien.

Puhl, R. (Hg.) 1997: Soziale Arbeit in Europa. Organisationsstrukturen, Arbeitsfelder und Methoden im Vergleich, Weinheim.

Ribhegge, H. 2007: Europäische Wirtschafts- und Sozialpolitik, Berlin.

Rieger, G. 2008: Europäische Sozialpolitik, in: Maelicke, B. (Hg.): Lexikon der Sozialwirtschaft, Baden-Baden, S. 316318.

Schendelen, R. von 2006: Die Champions League des Lobbying, in: Leif, T/Speth, R. (Hg.), S. 132-162.

Schendelen, R. von 2007: Trends im EU-Lobbying und in der EU-Forschung, in: Kleinfeld, R. u. a. (Hg.), Wiesbaden, S. 6591.

Schmid, J. 2002: Wohlfahrtstaaten im Vergleich. Soziale Sicherung in Europa: Organisation, Finanzierung, Leistungen und Probleme, Opladen.

Tömmel, I. (Hg.) 2007: Die Europäische Union. Governance and Policy-Making, PVS-Sonderheft 40, Wiesbaden.

Wessels, W. 2006: Das politische System der EU, in: Weidenfeld, W. (Hg.), S. 83-108.

Vahlpahl, T. 2007: Europäische Sozialpolitik, Wiesbaden. Weidenfeld, W. (Hg.) 2006: Die Europäische Union. Politisches System und Politikfelder, Berlin. 
„Verlief die Verständigung zwischen Politik und Sozialarbeit früher über Sprache, so sind jetzt Zahlen und Kennziffern gefragt. "

Hejo Manderscheid, Direktor des Diözesancaritasverbandes Limburg e. V.

"Keine Regierung und keine Bataillone vermögen Recht und Freiheit zu schützen, wo der Bürger nicht imstande ist, selber vor die Haustüre zu treten und nachzusehen, was es gibt."

Gottfried Keller, schweizerischer Schriftsteller (1819-1890)

„Folgen wir nur der Tradition, lebten wir

noch immer in Höhlen, folgten wir dem

Fortschritt, wäre dies bald wieder der Fall.."

Leszek Kolakowski, polnischer Philosoph (geb. 1927)

"Würdest du mir bitte sagen, wie ich von hier aus weitergehen soll? Das hängt zum großen Teil davon ab, wohin du möchtest, sagte die Katze."

Lewis Caroll, Alice im Wunderland (1865)

„Wenn wir Menschlichkeit wollen,

müssen wir menschliche Verhälthisse schaffen."

Bertolt Brecht, deutscher Schriftsteller (1898-1956)

"Freie Wohlfahrtspflege ist die Wohlfahrtspflege solcher Träger, die nicht Gebietskörperschaften sind und auch nicht deren Weisungen in Zweck- und Zielsetzung unterworfen sind."

Aus der Satzung der Bundesarbeitsgemeinschaft der Freien Wohlfahrtspflege e. V.

"Der Mensch ist von Natur ein Gemeinschaft bildendes Wesen." Aristoteles, griechischer Philosoph (384-322 v.Chr.) 